EON • Volume 14, Issue 4 - September 2021

\title{
ScholarOne and Editorial Manager: Tips and Tricks
}

\section{Miranda A. Sprouse ${ }^{1}$}

${ }^{1}$ Editoiral Assistant, J\&J Editorial

Published on: Sep 15, 2021

License: Creative Commons Attribution-NonCommercial-NoDerivatives 4.0 International License (CC-BY-NC-ND 4.0). 
Learning a new system requires quite a bit of patience and perseverance. There is so much information to absorb that it can be overwhelming at first, no matter what program you are learning to use. When I first started as an Editorial Assistant this past December, I knew that I would have to learn quickly to do my job properly. I was ready for it. But when I was introduced to two different systems instead of one, I will be the first to admit that it was daunting. It took a while to get my bearings and fully understand the tasks at hand.

In just the past six months, I have discovered a few tips and tricks that helped me with work in ScholarOne and Editorial Manager. Before jumping right in, I would like to disclose that there is still much I have to learn about both systems. My experience is limited; I only understand the basics of what I accomplish in my daily routine. However, I still strive to find ways to cut down on unnecessary time spent on tasks and improve my efficiency overall. My hope is that I can help provide ways for other ScholarOne and Editorial Manager users to improve their own efficiency as well.

ScholarOne is a very in-depth system. One element that I find truly helpful is the breakdown of the main action list into each individual step (Reviewer Selection, Make Decision, etc.). ScholarOne is very organized. Upon opening the manuscript submission, you will likely find most of the information you need on the first page. Although it can seem to be a lot of categories, data, and text, the system has a clear and comprehensive structure.

In comparison, Editorial Manager is much more concise. The "to-do" action lists on the homepage are minimal, with only a few key items to really focus on. Editorial Manager is not as visually intense as ScholarOne, but still has quite a bit of information for each manuscript that is not seen upfront. The color side bar is very helpful in determining importance: red takes priority, yellow needs attention sooner rather than later, and green is the last priority (these are usually the newest submissions). ScholarOne has color side bars as well, but these serve as indicators for different manuscript types.

Let us look at some tips and tricks for these two systems.

First and foremost, ProKeys. ProKeys is a free Google Chrome extension. It allows you to create shortcuts to replace a key word or phrase with a pre-written body of text. This tool is great for format check notes, email templates, or any text you find yourself 
frequently using. It works only on Internet browsers, but there are similar programs which can expand the shortcut to other applications. When I first started using it, I added my ProKeys snippet names next to their corresponding note so that I could familiarize myself with the shortcuts. In no time, I realized I hardly looked at my little cheat sheet anymore. I use ProKeys across all my journals but have found that I use it most frequently on ScholarOne's notepad. An example of one of my ProKeys: "colortext" and Shift + Space will replace "colortext" with my specific note to the authors asking them to ensure their text is in the default color. Overall, ProKeys is a major timesaver and has proven to be incredibly beneficial.

When working in Editorial Manager, one of my primary responsibilities is searching for blinding issues. The journals are double-blind, so anything that gives away any information about trials, authors, or author affiliations can prove to be a problem if left in the blinded manuscript. I have specific items to look for, usually found by searching for key phrases. For my first month or so, I opened all the files separately and searched each one, which took an unnecessary amount of time. Clicking "View Submission" in the action panel opens the full PDF with the title page, blinded manuscript, figures, tables, and references (if not configured to build into the PDF, supplemental materials must be opened separately). With the full PDF, I can easily scan and search for everything on my checklist in proper order, improving productivity. Every second that I can cut down on one manuscript is another second I can spend on the next submission.

Another potential time-waster is having to open and minimize several windows. When working in manuscript handling systems, a potential time-waster is having to open and manage several windows at once, such as when switching back and forth between protocol documents, notepads, figures, and other files. You can adjust the sizes of what you have open to make a split-screen, which cuts down the time spent looking for the window you need and opening it up again. If you have Windows, you can drag your Internet browser, picture, Word document, etc., to the side edge of your screen, where a transparent outline should pop up. This trick allows for you to perfectly split your screen with another window. No minimizing the screen and manually adjusting the size; this is quick and simple. You can also alter the split-screen if you choose to do so, whether you prefer one file to take up more space, or even have more documents on the screen (dragging windows into the corners will prompt them to take up a quarter of the space instead of half). If I am working on a format check, I like to have the manuscript on one side of my screen and the manuscript submission page (either on ScholarOne or Editorial Manager) on the other. I also have a separate monitor, where I 
split the screen with the protocol document and a notepad for quick ProKeys notes to copy into an email later.

One habit of mine, especially when I'm learning something new, is to jot quick notes down and re-write in a more coherent manner later. During my training, I scribbled away on my notepad noting how to tackle these new systems and little hints that my managers would provide. I re-created protocol documents pertaining to my specific assignments in ways that made more sense to me, which meant re-writing, reorganizing, and re-learning everything (not that the materials the team used were insufficient in any way, but that is how I think best). I made my own version of the format check documents, constructing it in a way that I felt would be the most advantageous to me.

For ScholarOne format checks for submitted manuscripts, I break it down into categories of submission details, title page, manuscript, figures, references, and extra files. I start with checking flags, manuscript type, iThenticate score, and any notes. From there, I look for journal-specific manuscript information (e.g., abstract, keywords, color text, headings, acknowledgments, supporting information paragraph). Then I re-scan the file for the figures, making sure nothing is against the journal guidelines, and finish up the manuscript by checking references for appropriate format. If the authors provide supplementary files, videos, permission forms, or cover art, I check these last.

Editorial Manager checks are similar, but I do not work with quite the same criteria. I also re-created a document with a revised order based on the action links: Details, File Inventory, and View Submission. The Details window includes author information, submission fees paid/unpaid/not required, submission questions, titles, and abstracts. The File Inventory window requires a quick scan, just to ensure blinding is present in file names, conflict of interest forms are included for each author, and I will open the manuscript file just to check the word count. Any keyword searches, figure checks, or overall scans can be completed by viewing the full submission PDF, which is the last step of my check.

The order, of course, can be reconfigured and altered to fit your own specifications. I believe it helps to structure the protocol documents in a way that works for you, because we all think very differently. While this specific tip is not necessarily for Editorial Manager or ScholarOne, it certainly helps to keep you organized and ensure that any work completed through the system has been done properly and effectively. 
These tips and tricks may not work for everyone. But hopefully they can provide a new perspective or inspire you to think creatively to improve your efficiency and job performance. As our workplaces and systems change and adapt, it never hurts to find different ways to expand and develop our performance.

\section{Disclosure Statement}

This author has no conflicts of interest to disclose.

\section{ISMTE Content - On Demand!}

Did you miss that session at last year's conference? Couldn't attend a recent webinar? Check out ISMTE's On-Demand Content for featured sessions that are free to access anytime, anywhere. Current highlights include Navigating International Communication in Scholarly Publishing, The Evolving World of Data Sharing, and Antiracism Toolkits for Allies in Scholarly Publishing! To browse available on-demand content, please visit https://www.ismte.org/page/ISMTE On-Demand. 